\title{
The High Field Magnet Laboratory at Radboud University Nijmegen
}

\author{
S.A.J. Wiegers • P.C.M. Christianen • \\ H. Engelkamp • A. den Ouden • \\ J.A.A.J. Perenboom · U. Zeitler · J.C. Maan
}

Received: 22 July 2009 / Accepted: 1 December 2009 / Published online: 30 December 2009

(C) The Author(s) 2009. This article is published with open access at Springerlink.com

\begin{abstract}
The High Field Magnet Laboratory is dedicated to materials research in the highest continuous fields, up to $33 \mathrm{~T}$, generated with resistive magnets. Circulating cold water evacuates the heat losses incurred when the coils are operating at a voltage drop of up to $500 \mathrm{~V}$ at the maximum current of $40 \mathrm{kA}$. There are three $20 \mathrm{MW}$ resistive magnets with bore sizes of 32 and $50 \mathrm{~mm}$, and a $50 \mathrm{~mm}$ bore hybrid magnet system allowing uninterrupted measurements at $30 \mathrm{~T}$ for many hours at a time. The infrastructure represents a major investment and has been in operation since 2003. At the moment HFML is extending its capabilities with the construction of a THz FEL.

In this paper we will describe the facilities and the possibilities to perform a wide range of experiments in materials research, and show some highlights of the research performed. Even higher fields can be made with a background field provided by a large-bore superconducting magnet: in this paper we will also present the construction of a hybrid magnet system that will generate fields to $45 \mathrm{~T}$.
\end{abstract}

Keywords Continuous magnetic fields · Hybrid magnet

\section{Introduction}

The High Field Magnet Laboratory of the Radboud University Nijmegen is operating a 20 MW installation in a new dedicated building since 2003 [1]. Much effort and costs were devoted towards optimizing the measurement conditions: the building is rugged and designed to minimize the coupling of vibrations to the user's probe, the access to the magnets is user-friendly with access on the same floor as well as from

S.A.J. Wiegers · P.C.M. Christianen · H. Engelkamp · A. den Ouden · J.A.A.J. Perenboom (凶) ·

U. Zeitler · J.C. Maan

High Field Magnet Laboratory, Institute for Molecules and Materials, Radboud University,

6525 ED Nijmegen, The Netherlands

e-mail: J.Perenboom@science.ru.nl 
a laboratory space on the floor above, the electrical power converters are designed for minimum ripple of the current (10 ppm), and operation of the installation is userfriendly and often under control of computers and the researchers only, with no need of operators.

The $20 \mathrm{MW}$ water cooling system was designed with a limited real-time cooling capacity of about $10 \mathrm{MW}$ (varying with atmospheric conditions) and a cold buffer of $1500 \mathrm{~m}^{3}$ water of between 8 and $10^{\circ} \mathrm{C}$.

\section{Improvement of the Cooling Installation}

The experience gained in running this installation for somewhat more than 5 years will allow us to further optimize the cooling installation. It was found that with the modes of operation chosen, the real-time cooling capacity falls seriously short of the design, partly because most of the time the warm water to be cooled can not be returned at the optimum temperature and flow. Also the minimum flow to the main heat exchangers of the magnet circuit is higher than needed to maintain the input temperature to the magnets, and this results in unnecessary load on the cold buffer and in returning cold water to the 'hot pool'. The consequence is that, although the cooling installation was designed to be able to run the magnets virtually without time restrictions when users would simply sweep the field (i.e. at an average power of 25-30\% of the magnet's maximum power uptake), that experiments often have to be terminated early and the researchers have to wait a few hours for the cold buffers to be restored.

By optimizing the cooling plant manually, and under moderate atmospheric conditions, i.e. when the wet bulb temperature does not exceed $15^{\circ} \mathrm{C}$ (in winter, spring and fall for the Netherlands), the real time cooling capacity exceeds $5,600 \mathrm{~kW}$ (mostly limited by the operation of the chillers), and this should be adequate for swept-field operation. In summer, when in the Netherlands the wet bulb temperature rarely rises above $20^{\circ} \mathrm{C}$, the cooling capacity is smaller, but the installation is still capable of producing water as cold as $10^{\circ} \mathrm{C}$. Experimenting with the cooling installation under realistic conditions, and analysis of its operation, has shown that with well-chosen modifications of the regulation of the installation significant improvements can be realized. The two most important issues are: better optimization of the flow to the main heat exchangers (by providing a by-pass and fast regulation); and using the two $750 \mathrm{~m}^{3}$ cold water buffers alternately, usually drawing the cold water from one while the other is cooled again by the cooling installation.

\section{The Hybrid Magnet Program}

We have installed a new $50 \mathrm{~mm}$ bore hybrid magnet to answer the needs of users who require more space. An existing hybrid magnet system was modified with a resistive insert based on the $50 \mathrm{~mm}$ bore resistive magnet, installed in 2007 [2], so producing a magnet system that generates $30 \mathrm{~T}$ with only $10 \mathrm{MW}$, and providing a useful and costefficient platform for researchers who also need the high field for extended periods, such as for NMR. 
Fig. 1 Schematic drawing of the $45 \mathrm{~T}$ hybrid magnet system, that will be constructed in Cell 6 of the HFML, and some of its auxiliary installations

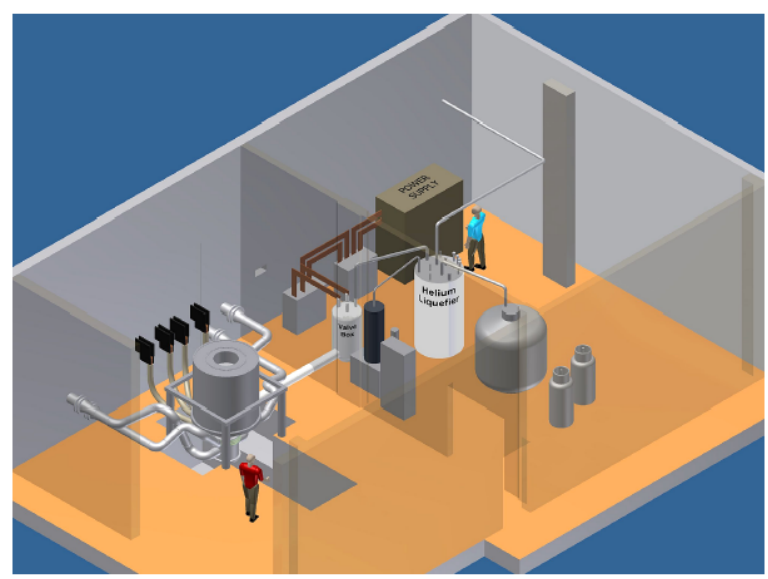

In addition (see Fig. 1), we are designing and constructing a $45 \mathrm{~T}$ hybrid system (with $32 \mathrm{~mm}$ bore). The $45 \mathrm{~T}$ hybrid magnet system will be based on a $600 \mathrm{~mm}$ clear bore, $12 \mathrm{~T}$ superconducting magnet, and will be placed in Cell 6 of the HFML. The superconducting coil will be all $\mathrm{Nb}_{3} \mathrm{Sn}$, with $10 \mathrm{kA}$ Cable-in-Conduit conductor in three different grades (high field, mid-field and low-field, with decreasing fraction of superconductor), and it will be operated in supercritical helium at $4.5 \mathrm{~K}$. The bore of the superconducting magnet will hold a $20 \mathrm{MW}$ resistive magnet, consisting of 5 nested coils, all designed and built with the Florida-Bitter technology, and largely based on high-strength Copper-Silver alloy plates.

The design and conductor test programs will be completed in 2010, and assembly of the hybrid magnet system on site will commence end of 2012, after fabrication of the materials and major components in industry. It is expected that the new $45 \mathrm{~T}$ hybrid magnet will be available for users in 2014 .

\section{Highlights of Research and Developments at HFML}

A myriad of experiments can be performed, such as magneto-spectroscopy from the visual down to the far-infrared and radio-frequencies, quantum oscillations as the de Haas-van Alphen (in magnetization) and Shubnikov-de Haas and Quantum Hall effects (in resistance), solid-state NMR MAS spectroscopy and electron-spin and cyclotron resonances, thermodynamic measurements as magnetization, specific heat, and thermopower, and often in a wide range of temperatures from room-temperature to sub Kelvin in using flow cryostats, bath cryostats, ${ }^{3} \mathrm{He}$ and ${ }^{3} \mathrm{He} /{ }^{4} \mathrm{He}$ dilution refrigerators.

More specifically, we have made great progress in fluorescence spectroscopy of single nano-objects (see Fig. 2) such as large molecules of biochemical interest and quantum dots. For these measurements the low vibration levels, and the stability of the sample cryostat with respect to the lasers and analysing spectrometers at the floor 
Fig. 2 Nano-object fluorescence spectroscopy probe with the 'attocube' $x y z$ sample positioner
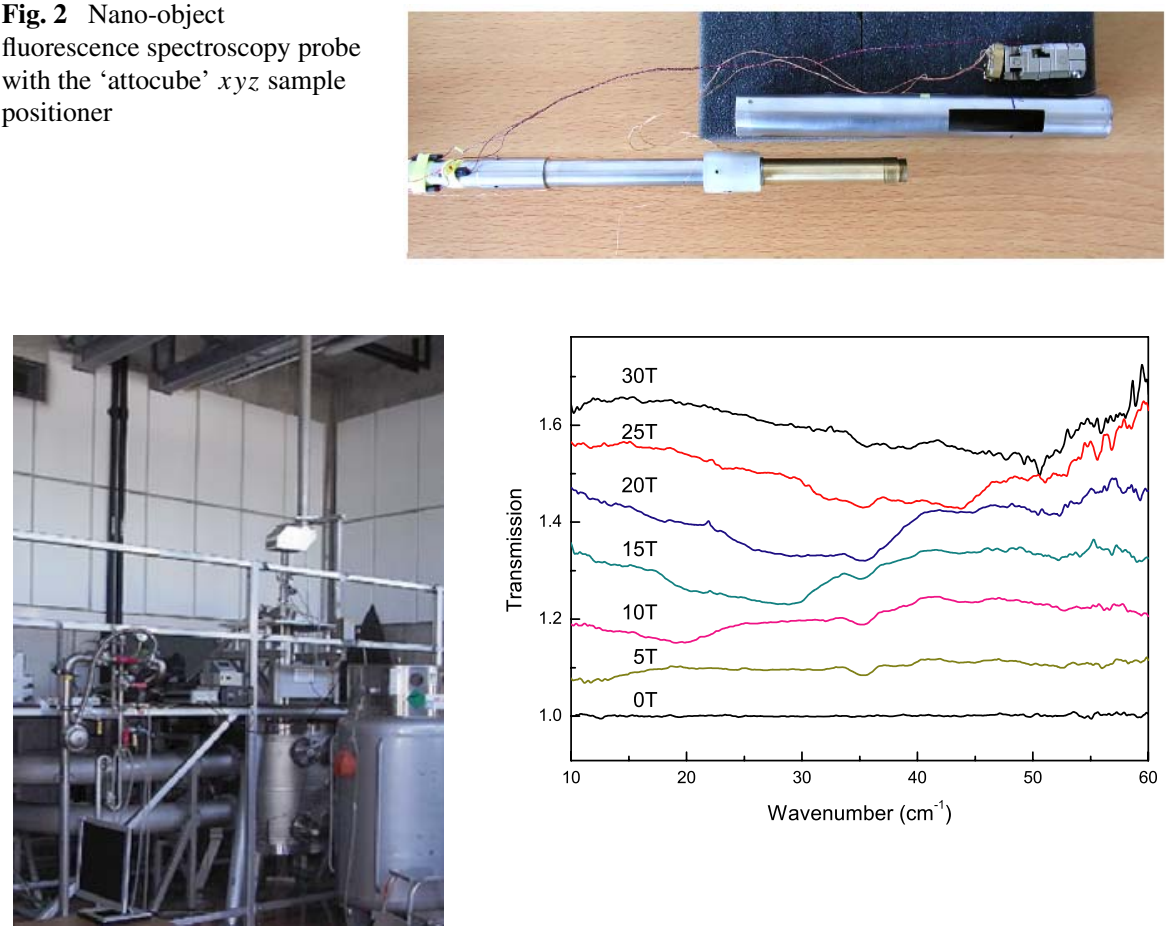

Fig. 3 Left: Coupling of far-infrared radiation from the Bruker interferometer to the sample cryostat using an evacuated beam line assembly; right: far infrared absorption of a cobalt-cluster compound showing the emergence with field and shift of electron spin resonances to $30 \mathrm{~T}$

above the magnet, were important enabling factors with the availability of 'attocube' piezo-driven sample positioners.

Far infrared spectroscopy will become one of the hot items at HFML with the cutting edge technology of the new $\mathrm{THz}$ free electron laser [3], and we have already made great progress in improved coupling of the weak far-infrared radiation of a Bruker FIR Fourier Transform Spectrometer with the sample in the measurement probe in the small bore of the magnet, see Fig. 3, already allowing far-infrared spectra to be taken in the $10-10,000 \mathrm{~cm}^{-1}$ range of wavenumbers, important for low-energy excitations in e.g. superconductors and magnetic materials.

Acknowledgements The operation of HFML and its user program is partly sponsored by the Stichting voor Fundamenteel Onderzoek der Materie (FOM) with financial support of the Netherlands Organisation for Scientific Research (NWO), and through the FP7 Infrastructures Program of the European Union contract 2280443, EuroMagNET.

Open Access This article is distributed under the terms of the Creative Commons Attribution Noncommercial License which permits any noncommercial use, distribution, and reproduction in any medium, provided the original author(s) and source are credited.

\footnotetext{
1 attocube systems AG, München, Germany (www.attocube.com).
} 


\section{References}

1. J.A.A.J. Perenboom, S.A.J. Wiegers, P.C.M. Christianen, U. Zeitler, J.C. Maan, J. Low Temp. Phys. 133, 181 (2003)

2. S.A.J. Wiegers, J. Rook, M.D. Bird, J. Toth, S. Bole, J.A.A.J. Perenboom, J.C. Maan, IEEE Trans. Supercond. 18, 564 (2008)

3. R.T. Jongma, W.J. van der Zande, A.F.G. der Meer, U. Lehnert, P. Michel, R. Wünsch, C.A.J. van der Geer, K. Dunkel, C. Piel, P.J.M. van der Slot, in Proceedings FEL'08, Gyeongju, Korea (2008), pp. 200-203 